\title{
Evaluation of Rubella, Toxoplasma gondii, and cytomegalovirus seroprevalences in women with miscarriage as adverse reproductive outcome in current pregnancy in ALBIYDA/LIBYA
}

\author{
kawakeb A.O.Saad ${ }^{1}$, Nagla Yousef ${ }^{2}$ \\ ${ }^{I}$ (Department of zoology, faculty of Sciences / Umar Al Mukhtar University, Libya) \\ ${ }_{2}^{2}$ (Department of zoology, faculty of Sciences / Umar Al Mukhtar University, Libya)
}

\begin{abstract}
This study aimed to determine the seroprevalence of Toxoplasma gondii, rubella, and cytomegalovirus among 71 pregnant women with age range from 19 to 45, who had mischarge as adverse reproductive outcome in current pregnancy in Albyda city, Libya. Peripheral blood samples were obtained from women how had mischarge recently for laboratory tests, IgM and IgG antibodies against $T$. gondii, rubella, and $C M V$ agents were detected by ELISA and results were expressed qualitatively as positive and negative. Among the tested sample Highest percentage was for IgG Rubella infection (98.59\%) followed by IgG CMV (96.36\%), and IgG toxoplasma (39.39\%). This study suggested that between the Rubella, Toxoplasma gondii, and cytomegalovirus infections during pregnancy cause the foetal loss.
\end{abstract}

Keywords: Cytomegalovirus; Rubella; toxoplasma; cytomigalo; Pregnancy.

\section{Introduction}

Abortion is an issue in pregnancy wastage with its concomitant social and economic [1]. Pregnancy loss has been attributed to several factors involved in human reproduction. Genetic and uterine abnormalities, endocrine and immunological dysfunctions, infectious agents, environmental pollutants, psychogenetic factors and endometriosis are most important causes of spontaneous abortion [2]. Infections caused by Toxoplasma gondii, Rubella virus and Cytomegalovirus are major causes of pregnancy lose in human. Women affected with any of these diseases during pregnancy are at high risk for miscarriage, stillbirth, or for a child with serious birth defects and/or illness. [3],[1].

Toxoplasma is a parasitic infectious disease caused by a protozoan Toxoplasma Gondii, which is transmitted to humans through the infection of food or water contaminated with cat faces or eating undercooked meat of the infected sheep, goat, cow, or pig and other avian species. The infection is carried to the infant through the mother's placenta, and can cause infections of the eyes or central nervous system. Identification of positive titer of immunoglobulin $\mathrm{G}(\mathrm{IgG})$ and immunoglobulin $\mathrm{M}(\mathrm{IgM})$ during pregnancy in women with previous negative titers of antitoxoplamsa $\operatorname{IgG}$ antibodies suggests a proliferative disease condition dangerous to the fetus and is more likely to cause a miscarriage or serious birth defects[4].

Rubella is caused by RNA virus of paramyxovirus group. It spreads mainly through family. Approximately 30\%-50\% fetuses of women who contact with Rubella during the first 3 months of pregnancy will be adversely affected by the virus. The Rubella virus readily invades the placenta and fetus during gestation [5].

In the case of Rubella, a woman in the first 2 or 3 months of pregnancy who is exposed may develop the infection and give birth to child with serious congenital defects such as deafness and blindness[6].

Cytomegalovirus (CMV): This infection is caused by DNA virus of herpes group. The common modes of infection are through saliva, urine, stool, breast milk, and unscreened blood transmission. Cytomegalovirus is a leading cause of congenital infections and long-term neurodevelopmental disabilities among children. High maternal sero-prevalence rates have been consistently associated with high congenital infection rates. Primary CMV infection in the mother results in a substantially higher risk of congenital CMV infection in the newborn (30\%-40\% risk) when compared with maternal CMV reactivation infection (1\%-3\% risk in the newborn) [7]. Fetal damage is more likely to be severe when maternal infection occurs early in pregnancy [8] . This study was and done primarily to calculate the seroprevalance of Toxoplasma gondii, Rubella and CMV infections among pregnant women with abortions as adverse reproductive outcome in current pregnancy.

\section{Material and methods}

This is a laboratory based study was done from June 2015 to May 2016, on patients who had attended the private clinic laboratory in ALBYDA city in LIBYA, after pregnancy lose or stillbirth. A total of 71 women in age ranged between 19-45 year old, were formed the study group. From each woman $3 \mathrm{ml}$ of venous blood was collected in a container with aseptic precaution. $\operatorname{IgM}$ and $\operatorname{IgG}$ antibodies against Toxoplasma goundii, 
Evaluation of Rubella, Toxoplasma gondii, and cytomegalovirus seroprevalences in women with..

Rubella and cytomegalovirus agents were detected by ELISA test kit, according to the manufacturer's instructions. Anti-Toxoplasma IgM antibody titres greater than a 0.490 index and anti-Toxoplasma IgG antibody titres greater than $3.0 \mathrm{IU} / \mathrm{ml}$ were considered positive. Anti-rubella IgM antibody titres greater than 0.600 and anti-rubella IgG antibody titres greater than $10.0 \mathrm{IU} / \mathrm{ml}$ were considered positive. Anti-CMV IgM antibody titres greater than 0.500 and anti-CMV IgG antibody titres than 15.0 AU/ml were considered positive. Results were expressed qualitatively as positive and negative. In case of equivocal result, test was repeated on fresh sample and if still equivocal, it was not included in data analysis.

\section{Results}

Overall prevalence of toxoplasma, Rubella and MCV IgG, and IgM antibodies in pregnant women who had mischarge with recent pregnancy, was high for anti-Rubella IgG (98.59\%), 70 women out of 71, followed by anti-CMV IgG (95.77\%), 68 women out of 71 and anti-toxoplasma IgG was (39.43\%), 28 women out of 71 cases, while IgM anti-Rubella, anti-toxoplasma and anti-CMV were $(0 \%),(0 \%)$ and $(2.81 \%)$ respectively (Table 1). IgG antibodies towards toxoplasma was seen with rubella in 28 cases (39\%), toxoplasma and CMV in 28 cases (39\%), toxoplasma, rubella and CMV was seen in 28 cases (39\%),rubella and CMV together in 67 cases (94\%) (Table 2)

Table 1: $\operatorname{IgG}$ and IgM seropositivity in 71 cases of pregnancy lose

\begin{tabular}{|c|c|c|c|c|c|}
\hline Test & Negative (n) & $\%$ & Positive (n) & $\%$ & Total \\
\hline Anti-Rubella IgM & 71 & 100 & 0 & 0 & 71 \\
\hline Anti-Rubella IgG & 1 & 1.40 & 70 & 98.59 & 71 \\
\hline Anti-Toxoplasma IgM & 71 & 100 & 0 & 0 & 71 \\
\hline Anti-Toxoplasma IgG & 43 & 60.56 & 28 & 39.43 & 71 \\
\hline Anti-CMV IgM & 69 & 97.18 & 2 & 2.81 & 71 \\
\hline Anti-CMV IgG & 3 & 4.22 & 68 & 95.77 & 71 \\
\hline
\end{tabular}

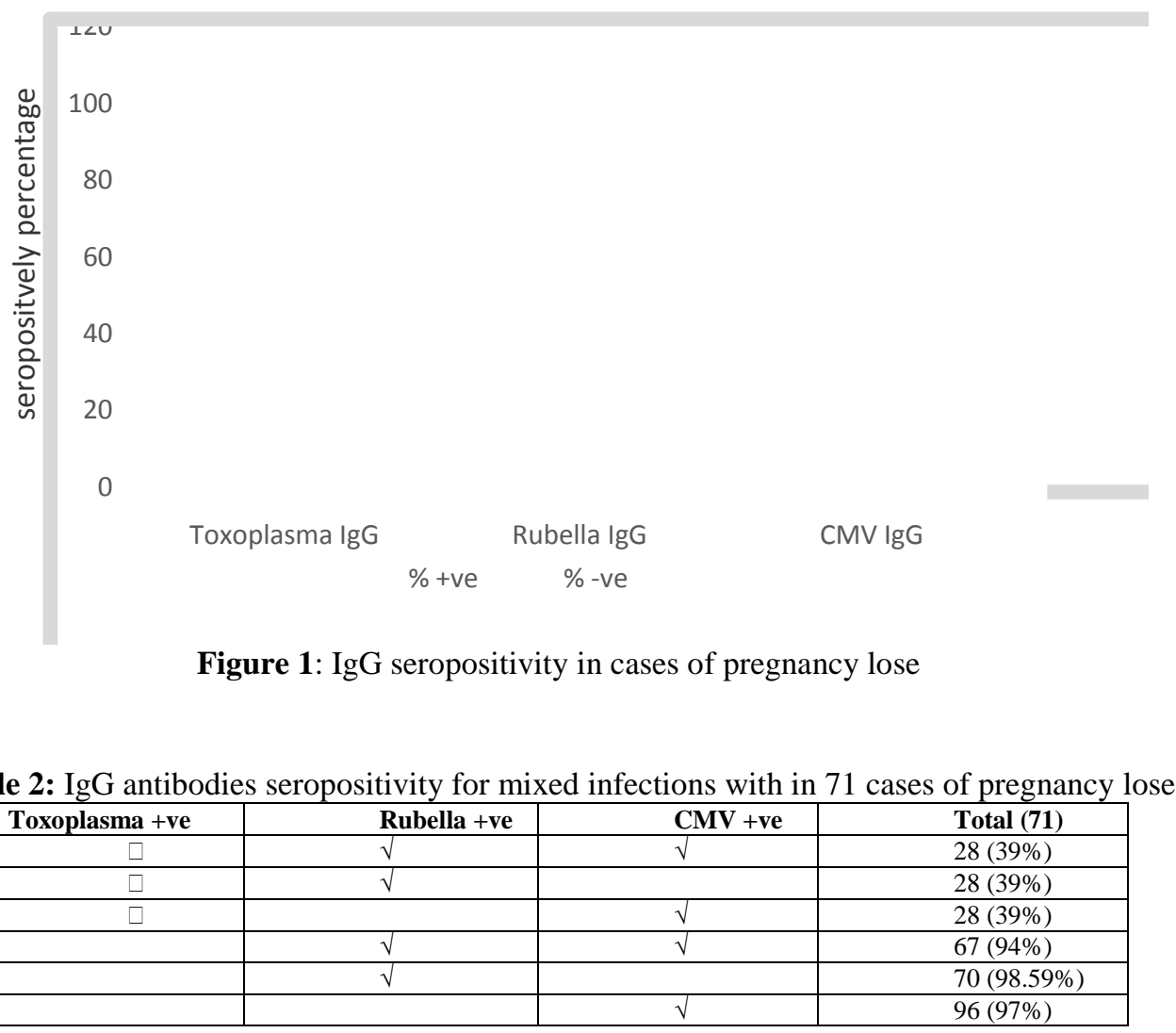

\section{Discussion}

Rubella, toxoplasma and cytomegalo agents are known to cause infections in utero and are often responsible for pregnancy lose. But there are considerable geographical variations in the prevalence of these agents among the women of child bearing age and are responsible for pregnancy wastage[5]. the current study 
found that prevalence of $\operatorname{IgG}$ Rubella infection (98.59 \%), $\operatorname{IgG}$ CMV (96.36\%), and $\operatorname{IgG}$ toxoplasma (39.39\%).Parlak,2015 in Turkey, have also reported overall Anti-Toxoplasma, anti-Rubella, and anti-CMV IgM and $\operatorname{IgG}$ antibody positivity rates were $1.1 \%, 0.5 \%$ and $2.6 \%$, and $37.6 \%, 86.5 \%$ and $100 \%$, respectively [9].

This study revealed that the prevalence of Rubella and CMV in pregnant women are very high, antiRubella IgG and anti-CMV IgG antibodies were found in $(98.5 \%)$ and $(95.8 \%)$ of the cases respectively, while anti-Rubella IgM and anti-CMV IgM antibodies were $(0 \%)$ and $(2.8 \%)$ of the subjects tested positive respectively. The detection of IgG indicated that the pregnant women had previously been infected with Rubella and CMV viruses. After the infection, IgG remains in the body for life and protects considerably against the next infections.

There are many studies about the association between CMV infection and pregnancy loss; these studies showed that CMV can result in abortion or stillbirth [10, 11]. CMV infection in pregnancy has a higher incidence of symptomatic congenital infection and foetal loss[12]. seroprevalance of CMV IgG observed in current study is agree with some other studies in different other countries in the same region, (Nahla KH 2011) reported that CMV IgG in pregnant women was (98.3\%) [13], 96\% in Egypt [14]and 97.2\% in Nigeria [15], in Iran (96\%) [16], Saudi Arabia (91.1\%) ([17], across the globe, that more than one lac children are born with congenital rubella syndrome each year, most of them in developing countries[18].

The prevalence of anti-toxoplasma antibodies was found in our study lower than that found in Benin 53.6\% [19]; 40.2\% in Dakar, Senegal[20]; and 34.1\% from pregnant women in Sudan [21]. However, it is higher than the prevalence of $12.8 \%$ observed among pregnant women in South Africa [22]. All these previous studies confirmed that, congenital infections with $\mathrm{T}$. gondii and rubella are a significant cause of neonatal mortality and infant morbidity in the world. Therefore more research with higher samples is required for clarification of the role of toxoplasmosis in etiology of abortion and stillbirth in Libya. Primary prevention of toxoplasmosis in the seronegative pregnant mother can be achieved through education to practice precautionary measures, which include washing the hands frequently, washing all vegetables and fruits and, most importantly, avoidance of eating raw meat. We should build public health strategies to educate women about the prevention of these infections.

\section{Acknowledgements}

I would like to thank the MECA Laboratories technicians who provided useful and helpful assistance.

\section{References}

[1]. Tiwari, S., B.S. Arora, and R. Diwan, TORCH IgM seroprevalence in women with abortions as adverse reproductive outcome in current pregnancy. International Journal of Research in Medical Sciences, 2016. 4(3): p. 784-788.

[2]. Dicker D, G.J., Levy T, Feldberg D, Ashkenazi J., Theimpact of long-term gonadotropin-releasing hormone analoguetreatment on preclinical abortion in patients with severe endometriosisundergoing in vitro fertilization-embryo transfer. Fertil. Steril, 1992. 57: p. 597-600.

[3]. Simgamsetty, S., et al., Study of seroprevalance of Toxoplasma gondii, Rubella virus and Cytomegalovirus (ToRC) infections in antenatal women presented with bad obstetric history and comparative evaluation of Nanoplex ToRCH screen ELISA kit with VIDAS. Int J Res Med Sci, 2015. 3(5): p. 1203-1208.

[4]. Gomella, T.L., Infectious Diseases: TORCH Infections. In Neonatology: Management, Procedures, On-Call Problems, Diseases and Drugs, . Norwalk, CT: Appleton \&Lange Appleton \&Lange, 1994.

[5]. Coulter, C., R. Wood, and J. Robson, Rubella infection in pregnancy. Communicable diseases intelligence, 1999. 23: p. 93-96.

[6]. Deorari AK, B.S., Maitreyi RS, Agarwal D, Kumar H, Paul VK, et al, Incidence, clinical spectrum, and outcome of intrauterine infections in neonates. Journal of Tropical Pediatrics., 2000. 46(3): p. 155-9.

[7]. Stagno S, P.R., Dworsky ME, Henderson RE, Moore EG, Walton PD, et al. , Congenital cytomegalovirus infection: the relative importance of primary and recurrent maternal infection. The New England Journal of Medicine, 1982. 306(16): p. 945-9.

[8]. Boppana SB, Pass RF, and B. WJ., Virus-specific antibody responses in mothers and their newborn infants with asymptomatic congenital cytomegalovirus infections. The Journal of Infectious Diseases., 1993. 167(1): p. 72-7.

[9]. Parlak, M., et al., Seroprevalence of Toxoplasma, Rubella, and Cytomegalovirus among pregnant women in Van. Obstet Gynecol, 2015. 2: p. 79-82.

[10]. Fairley, J., et al., Human cytomegalovirus infection inhibits epidermal growth factor (EGF) signalling by targeting EGF receptors. Journal of General Virology, 2002. 83(11): p. 2803-2810.

[11]. Nahum, A., et al., The biological significance of TLR3 variant, L412F, in conferring susceptibility to cutaneous candidiasis, CMV and autoimmunity. Autoimmunity reviews, 2012. 11(5): p. 341-347.

[12]. Turbadkar, M., Rele., Seroprevalence of TORCH infections in bad obstetric history. . Indian J of Med Micro, 2003. 21: p. 108-10.

[13]. Nahla KH, A.Y., Enan KH . Studies on congenital infections in Sudan: Seroprevalence of cytomegalovirus. . J. Sci. Tech. , 2011. 12(4): p. 82-90.

[14]. El-Nawawy, S., El Azzouni, Amer El-S, Karim MA, Demian S, El Sayed M Maternal and neonatal prevalence of toxoplasma and cytomegalovirus (CMV) antibodies and hepatitis-B antigens in an Egyptian rural area. J. Trop. Pediatr, 1996. 42(3): p. $154-157$.

[15]. Akinbami , R., Adewunmi , Wright, Dosunmu, Adeyemo, Adediran, Osunkalu . Seroprevalence of cytomegalovirus antibodies amongst normal pregnant women in Nigeria. Int. J. W. H, 2011. 3: p. 423-428.

[16]. Doroudchi M, et al., Seroepidemiological survey of rubella immunity among three populations in Shiraz, Islamic Republic of Iran. East Meditterr. Health J. , 2001. 7 p. 128-38.

[17]. Hossain, A., Seroepidemiology of rubella in Saudi Arabia. J. Trop. Pediatr, 1989. 35: p. 169-170.

[18]. Vijayalakshmi, A.R., Prakash K Narendran K, Ravindran M, Prajna L et al. , Rubella serosurveys at three Aravind eye hospitals in Tamil Nadu, India. Bull World Health Organ., 2004. 82: p. 259-64. 
Evaluation of Rubella, Toxoplasma gondii, and cytomegalovirus seroprevalences in women with..

[19]. Rodier, M.H., et al., Seroprevalences of toxoplasma, malaria, rubella, cytomegalovirus, HIV and treponemal infections among pregnant women in Cotonou, Republic of Benin. Acta Tropica, 1995. 59(4): p. 271-277.

[20]. Faye, O., et al., La toxoplasmose a Dakar. Bull Soc Pathol Exot, 1998. 91: p. 249-50.

[21]. Elnahas, A., et al., Toxoplasmosis in pregnant Sudanese women. Saudi medical journal, 2003. 24(8): p. 868-870.

[22]. Kistiah, K., et al., Seroprevalence of Toxoplasma gondii infection in HIV-positive and HIV-negative subjects in Gauteng, South Africa. Southern African Journal of Epidemiology and Infection, 2011. 26(4): p. 225-228. 